DE

M E D I C I N A

T R O P I C A L

$\mathrm{DE}$

SÃO PAULO

JOURNAL OF THE SÃO PAULO INSTITUTE OF TROPICAL MEDICINE

${ }^{1}$ Instituto Butantan, Divisão de Ensaios Clínicos e Farmacovigilância, São Paulo, São Paulo, Brazil

2Universidade de São Paulo, Faculdade de Medicina, Departamento de Pediatria, São Paulo, São Paulo, Brazil

Correspondence to: Vera Lúcia Gattás Instituto Butantan, Divisão de Ensaios Clínicos e Farmacovigilância, Av. Vital Brazil, 1500, CEP 05503-900, São Paulo, SP, Brazil

Tel: +55 11 3723-2126

E-mail: vera.gattas@ butantan.gov.br

Received: 15 August 2018

Accepted: 27 November 2018

\section{Safety assessment of seasonal trivalent influenza vaccine produced by Instituto Butantan from 2013 to 2017}

Vera Lúcia Gattás', Patrícia Emília Braga1, Marcelo Eiji Koike', Maria Beatriz Bastos Lucchesi ${ }^{1}$, Mayra Martho Moura de Oliveira ${ }^{1}$, Roberta de Oliveira Piorelli' ${ }^{1}$, Vivian Queiroz', Alexander Roberto Precioso ${ }^{1,2}$

\section{ABSTRACT}

Vaccination has been a successful strategy in influenza prevention. However, despite the safety and efficacy of the vaccines, they can cause adverse events following immunization (AEFI). Moreover, due to the vaccination success, most of vaccine-preventable diseases (VPD) have become rare, and public attention has been shifted from VPD to the AEFI associated with vaccination. This manuscript describes the safety of Instituto Butantan (IB) seasonal trivalent influenza vaccine (TIV) from 2013 to 2017. AEFI data were received by the Department of Pharmacovigilance of IB (PV-IB), from January the $1^{\text {st }} 2013$ to December the $31^{\text {st }} 2017$, and were recorded in an electronic database $\left(\right.$ OpenClinica $\left.{ }^{\odot}\right)$. PV-IB received 1,415 Individual Case Safety Reports (ICSR) associated with the TIV; 1,253 ICSR with at least one AEFI were analyzed and 4,140 AEFI were identified. The other $162(11.4 \%)$ cases did not present any symptom. Among the total of AEFI, 405 (9.8\%) were classified as serious. AEFI with the highest incidence rates per 100,000 doses of TIV were: "local pain" (0.28), "local erythema" (0.23), "local warmth" (0.22), "local swelling" $(0.20)$ and "fever" (0.19). PV-IB received 175 (4.2\%) occurrences of SAE of special interest, of which 75 (1.8\%) anaphylaxis/anaphylactic reactions, $56(1.4 \%)$ neurological syndromes (including seven Guillain-Barré Syndrome) and 44 (1.1\%) convulsion/febrile convulsion. The results of this manuscript suggested that Instituto Butantan trivalent influenza vaccine (IB-TIV) is safe, as most of the reported AEFI were classified as non-serious. AEFI described for the IB-TIV are in agreement with the ones described in the literature for similar vaccines.

KEYWORDS: Influenza vaccine. Adverse events following immunization (AEFI). Safety vaccines. Surveillance. Instituto Butantan.

\section{INTRODUCTION}

Seasonal influenza is an acute respiratory infection caused by three types of seasonal influenza viruses, A, B, and C, which have global circulation. Influenza type $A$ and $B$ viruses have more clinical significance while Influenza type $C$ virus is much less frequent and usually causes mild infections, presenting less significant public health implications. Due to this reason, influenza vaccines are produced using relevant strains of influenza $\mathrm{A}$ and $\mathrm{B}$ viruses ${ }^{1}$.

The prevention of influenza

Since the 1940s, the vaccination has been a successful strategy in influenza prevention. However, despite the safety and efficacy of the vaccines, they can 
cause some adverse events following immunization (AEFI). Moreover, due to the success of vaccines, most of vaccine-preventable diseases (VPD) have become rare, and public attention has been shifted from VPD to some adverse events associated with vaccination. Because of this, the pharmacovigilance (PV) is an important tool to monitor AEFI and confirm the benefits from immunization in different target groups such as infants, children, elderly and others ${ }^{2}$.

\section{The influenza vaccine in Brazil}

Since 1999, influenza vaccination campaigns have been conducted annually in Brazil, targeting the groups at higher risk for influenza complications, that are children, elderly and those with chronic diseases (pulmonary or cardiovascular diseases, immunocompromised, transplant receptors, among others) ${ }^{3}$.

Over the last five years (2013-2017), the National Immunization Campaigns reached vaccine coverage rates ranging from $86.8 \%$ to $94.4 \%{ }^{3}$. In 2001, the Brazilian government signed a technology transfer agreement between Instituto Butantan of Sao Paulo and Sanofi-Pasteur/ Aventis. In the 2013 season, the seasonal influenza vaccine produced by Instituto Butantan began to be delivered to the Brazilian Ministry of Health (MoH). From 2013 to 2017, $\mathrm{MoH}$ received 158,735,729 doses of influenza vaccine from Instituto Butantan. Since the first distributed dose, the Pharmacovigilance Department of Instituto Butantan (PV-IB) has been conducting routine activities to monitor the adverse events associated with the vaccine according to the Brazilian Health Regulatory Agency (Anvisa) 4 . As part of PV-IB routine activities, we conducted the analysis of the AEFI associated with the seasonal trivalent influenza vaccine (split virion, inactivated) produced by Instituto Butantan from 2013 to 2017.

\section{METHODS}

\section{Study design}

Descriptive analysis of individual case safety reports (ICSR) of adverse events following immunization (AEFI), immunization errors and pregnant women exposed to the seasonal trivalent influenza vaccine (TIV) manufactured by Instituto Butantan.

\section{Data collection}

The ICSR were received from different sources of notification by the Pharmacovigilance Department of
Instituto Butantan (PV-IB), and were recorded in an electronic database $\left(\right.$ OpenClinica $\left.{ }^{\odot}\right)$.

\section{Study period}

The period of analysis was from January the $1^{\text {st }} 2013$ to December the $31^{\text {st }} 2017$.

\section{Study variables}

A notification form was completed for each report with the following information: reporter occupation, vaccinee socio-demographic characteristics, adverse events signs and symptoms, clinical outcomes, year of vaccination, occurrence of immunization errors and vaccine exposure during pregnancy. All AEFI were coded according to Medical Dictionary for Regulatory Activities (MedDRA), using the preferred term (PT) and its allocation by system organ class (SOC) $)^{5}$.

\section{Sources of notification}

The AEFI data, vaccine exposure during pregnancy and immunization errors were identified by PV-IB from the following sources of notification:

1) Spontaneous reports: data reported by Health Care Professionals and vaccinees from the public health services directly to PV-IB;

2) National Immunization Program of the Brazilian Ministry of Health (NIP): data reported by the public health services to NIP through the National Surveillance System and forwarded to PV-IB;

3) Active Pharmacovigilance (Active PV): data of pregnant women exposed to TIV presenting or not AEFI during a Post-Authorization Safety Study (PASS) conducted by PV-IB in 2017 National Immunization Campaign (ClinicalTrials.gov registry N ${ }^{\circ}$ NCT03057483).

\section{Definitions}

1) Adverse Event Following Immunization (AEFI): any untoward medical occurrence affecting a vaccinated person and that does not necessarily have a causal relationship to the vaccine. Therefore, an adverse event can be any unfavorable and unintended sign, symptom, or disease (including an abnormal laboratory finding), temporally associated with the vaccination ${ }^{6}$;

2) Individual Case Safety Report (ICSR): any report of an AEFI that has occurred in a patient taking one or more medicines ${ }^{7}$. A report of medicine exposure during pregnancy or immunization error also constitutes an ICSR; 
3) Serious Adverse Event (SAE): any AEFI that results in death, hospitalization or prolongation of hospitalization, life-threatening, congenital anomaly or birth defect and persistent or significant disability. A medically significant AEFI (i.e., an adverse event arising from the use of medicines requiring medical intervention in order to avoid death, life-threatening, significant disability or hospitalization) was considered as a SAE. The other AEFI were considered non-serious (NSAE) ${ }^{6}$;

4) Serious Adverse Events of Special Interest (SAESI): all of the following SAE were considered SAESI: anaphylaxis/anaphylactic reaction, convulsion/febrile convulsion, neurological syndromes (e.g., Guillain-Barré Syndrome (GBS) encephalomyelitis and neuropathy/ neuritis), vasculitis and thrombocytopenia. All cases of GBS were analyzed for their causal association with the vaccination and clinical outcomes of the individuals ${ }^{8}$.

\section{Data analysis}

Data analysis was performed using the statistical software STATA $^{\oplus}$, version 13.0 (StataCorp LP, College Station, Texas, USA) and data were analyzed by year of vaccination. The results are presented as frequencies of occurrence.

AEFI incidence rates were calculated by dividing the number of adverse events, per year of vaccination, by the number of distributed doses of Instituto Butantan TIV. We considered one administered dose the same as one distributed dose since NIP has not made the number of administered dose per vaccine manufacturer publicly available.

Additionally, cases of vaccine exposure during pregnancy were analyzed according to the occurrence of AEFI over the entire study period. Post-licensing monitoring of pregnant women exposed to the vaccine was done as the World Health Organization (WHO) considers it a relevant safety monitoring that should be included in the pharmacovigilance activities of Marketing Authorization Holders (MAH) ${ }^{9}$.

\section{Ethics statement}

All data analyzed in this manuscript came from the pharmacovigilance activities, which are mandatory for MAH, according to legal marks established by Anvisa ${ }^{4}$. Regarding the Active PV the Research Ethics Committee approved the study (approval $\mathrm{N}^{\circ} 2.034 .906$ ) and the enrolled pregnant participants signed an Informed Consent Form authorizing their data submission to PV-IB to follow them up during the postpartum period.

\section{RESULTS}

Individual case safety reports

From 2013 to 2017, the PV-IB received 1,510 ICSR associated with the seasonal trivalent influenza vaccine (TIV) manufactured by Instituto Butantan. Of this total, 95 (6.3\%) ICSR were excluded from the analysis because they did not present the year of vaccination and PV-IB could not ensure that vaccinations occurred during the period under review.

Considering all the valid cases received (1,415), 222 (15.7\%) were reported in 2013, $247(17.5 \%)$ in 2014, 708 $(50.0 \%)$ in 2015, $71(5.0 \%)$ in 2016 and $167(11.8 \%)$ cases in 2017.

The majority of the ICSR occurred in female vaccinees $(875=61.8 \%)$ and in the age group under 10 years old $(695=49.1 \%)$ (Table 1$)$.

Among the total of ICSR $(1,415)$ there were 1,253 (88.6\%) with at least one symptom (NSAE or SAE). The other 162 $(11.4 \%)$ cases did not present any symptom, however they were analyzed since they were cases of pregnant women exposed to the vaccine or cases of immunization error.

Most of ICSR with at least one symptom received during the period covered in this analysis, was classified as non-serious, representing $891(71.1 \%)$ of the individual cases, while 362 (28.9\%) were classified as serious due to an occurrence of at least one SAE.

\section{Adverse events following immunization}

From 2013 to 2017 , among the 1,253 ICSR 4,140 AEFI were reported: 487 (11.8\%) in 2013, 839 (20.3\%) in $2014,2,384(57.6 \%)$ in 2015,198 (4.8\%) in 2016 and 232 $(5.6 \%)$ in 2017.

In order to calculate AEFI incidence rates, it is important to mention that Instituto Butantan provided to $\mathrm{MoH}$ $158,735,729$ doses of TIV of which $6,130,200$ doses in $2013,10,280,270$ doses in 2014, 46,170,040 doses in 2015, 51,154,819 doses in 2016 and 45,000,400 doses in 2017. AEFI with the highest incidence rates per 100,000 doses of TIV were: "local pain" (0.28), "local erythema" (0.23), "local warmth" (0.22), "local swelling" (0.20) and "fever" (0.19) (Table 2).

Regarding the seriousness of the 4,140 AEFI, 405 (9.8\%) were classified as SAE. Among the total of SAE (405), PV-IB received 175 (43.2\%) notifications of SAESI, of which 75 (18.5\%) anaphylaxis/anaphylactic reactions, 56 (13.8\%) neurological syndromes (including seven GuillainBarré Syndrome) and 44 (10.9\%) were convulsion/febrile convulsion. 
Table 1 - Distribution of ICSR† according to source, reporter type, social demographic characteristics and year of vaccination. Brazil, 2013-2017.

\begin{tabular}{|c|c|c|c|c|c|c|c|c|c|c|c|c|}
\hline \multirow{3}{*}{ Characteristics } & \multicolumn{12}{|c|}{ Year of vaccination } \\
\hline & \multicolumn{2}{|c|}{$\begin{array}{c}2013 \\
(n=222)\end{array}$} & \multicolumn{2}{|c|}{$\begin{array}{c}2014 \\
(n=247)\end{array}$} & \multicolumn{2}{|c|}{$\begin{array}{c}2015 \\
(n=708)\end{array}$} & \multicolumn{2}{|c|}{$\begin{array}{l}2016 \\
(n=71)\end{array}$} & \multicolumn{2}{|c|}{$\begin{array}{c}2017 \\
(n=167)\end{array}$} & \multicolumn{2}{|c|}{$\begin{array}{c}\text { Total } \\
(\mathrm{n}=1,415)\end{array}$} \\
\hline & $\mathrm{n}$ & $\%$ & $\mathrm{n}$ & $\%$ & $\mathrm{n}$ & $\%$ & $\mathrm{n}$ & $\%$ & $\mathrm{n}$ & $\%$ & $\mathrm{n}$ & $\%$ \\
\hline \multicolumn{13}{|l|}{ Source } \\
\hline Spontaneous & 56 & 25.2 & 63 & 25.5 & 30 & 4.2 & 60 & 84.5 & 58 & 34.7 & 267 & 18.9 \\
\hline NIP $\ddagger$ & 166 & 74.8 & 184 & 74.5 & 678 & 95.8 & 11 & 15.5 & 3 & 1.8 & 1,042 & 73.6 \\
\hline Active PV\# & 0 & 0 & 0 & 0 & 0 & 0 & 0 & 0 & 106 & 63.5 & 106 & 7.5 \\
\hline \multicolumn{13}{|l|}{ Reporter type } \\
\hline HCP§ & 194 & 87.4 & 199 & 80.6 & 697 & 98.4 & 51 & 71.8 & 135 & 80.8 & 1,276 & 90.2 \\
\hline Non-HCP§ & 24 & 10.8 & 45 & 18.2 & 9 & 1.3 & 16 & 22.5 & 21 & 12.6 & 115 & 8.1 \\
\hline Not informed & 4 & 1.8 & 3 & 1.2 & 2 & 0.3 & 4 & 5.6 & 11 & 6.6 & 24 & 1.7 \\
\hline \multicolumn{13}{|l|}{ Sex } \\
\hline Female & 126 & 56.8 & 160 & 64.8 & 413 & 58.3 & 38 & 53.5 & 138 & 82.6 & 875 & 61.8 \\
\hline Male & 90 & 40.5 & 78 & 31.6 & 293 & 41.4 & 31 & 43.7 & 23 & 13.8 & 515 & 36.4 \\
\hline Not informed & 6 & 2.7 & 9 & 3.6 & 2 & 0.3 & 2 & 2.8 & 6 & 3.6 & 25 & 1.8 \\
\hline \multicolumn{13}{|l|}{ Age (years) } \\
\hline $0-4$ & 66 & 29.7 & 108 & 43.7 & 305 & 43.1 & 36 & 50.7 & 11 & 6.6 & 526 & 37.2 \\
\hline $5-9$ & 14 & 6.3 & 14 & 5.7 & 137 & 19.4 & 0 & 0 & 4 & 2.4 & 169 & 11.9 \\
\hline $10-19$ & 6 & 2.7 & 6 & 2.4 & 25 & 3.5 & 2 & 2.8 & 12 & 7.2 & 51 & 3.6 \\
\hline $20-29$ & 17 & 7.7 & 13 & 5.3 & 37 & 5.2 & 4 & 5.6 & 80 & 47.9 & 151 & 10.7 \\
\hline $30-39$ & 28 & 12.6 & 17 & 6.9 & 49 & 6.9 & 8 & 11.3 & 25 & 15.0 & 127 & 9.0 \\
\hline $40-49$ & 19 & 8.6 & 15 & 6.1 & 39 & 5.5 & 4 & 5.6 & 8 & 4.8 & 85 & 6.0 \\
\hline $50-59$ & 24 & 10.8 & 7 & 2.8 & 37 & 5.2 & 5 & 7.0 & 3 & 1.8 & 76 & 5.4 \\
\hline $60-69$ & 25 & 11.3 & 10 & 4.0 & 42 & 5.9 & 3 & 4.2 & 8 & 4.8 & 88 & 6.2 \\
\hline$\geq 70$ & 10 & 4.5 & 12 & 4.9 & 33 & 4.7 & 2 & 2.8 & 6 & 3.6 & 63 & 4.5 \\
\hline Not informed & 13 & 5.9 & 45 & 18.2 & 4 & 0.6 & 7 & 9.9 & 10 & 6.0 & 79 & 5.6 \\
\hline
\end{tabular}

†ICSR: Individual Case Safety Report; ‡NIP: National Immunization Program; \#PV: Pharmacovigilance; §HCP: Health Care Professional

It is important to emphasize that among the total of SAE only $3(0.7 \%)$ were assessed for causality as "certainly related" to the vaccine: arrhythmia, hypersensitivity and laryngeal edema.

Considering that GBS is a rare and closely monitored SAE by health authorities, it is noteworthy that three cases were assessed as "probably related" and one case was "possibly related" to the vaccine. The other three cases could not be assessed for causality due to insufficient information (diagnosis and medical history lack of information).

Regarding the outcome of the "probably related" GBS, in one case full recovery was achieved, in another case the outcome was not reported and in the remaining case the individual evolved to death. The death occurred in an elderly vaccinee who was diagnosed with GBS while undergoing treatment for herpes zoster. The onset interval between vaccination and the first symptom was one month.
The outcome of the "possibly related" GBS was death. This SAESI occurred in a vaccinee who had diabetes mellitus (type 2), systemic arterial hypertension, glaucoma and obesity and it was not possible to confirm whether death was caused by the worsening of health conditions or by the GBS. In respect to the three cases that could not be assessed for causality, one recovered without sequelae and two did not have the outcomes reported.

A total of 388 SAE could not be assessed for causality due to insufficient information on the correct diagnosis and the medical history.

\section{Pregnancy}

Over the descriptive analysis period, PV-IB received 155 notifications of pregnant women exposed to Instituto Butantan TIV, of which 106 (68.4\%) from the Active PV 
Table 2 - Incidence rates for AEFI† with the influenza vaccine according to administered doses. Brazil, 2013-2017.

\begin{tabular}{|c|c|c|c|c|c|c|c|c|c|c|c|c|}
\hline \multirow{4}{*}{ AEFI† } & \multicolumn{12}{|c|}{ Year of vaccination } \\
\hline & \multirow{2}{*}{\multicolumn{2}{|c|}{$\frac{2013}{(n=487)}$}} & \multirow{2}{*}{\multicolumn{2}{|c|}{$\frac{2014}{(n=839)}$}} & \multirow{2}{*}{\multicolumn{2}{|c|}{$\begin{array}{c}2015 \\
(n=2,384)\end{array}$}} & \multirow{2}{*}{\multicolumn{2}{|c|}{$\begin{array}{c}2016 \\
(n=198)\end{array}$}} & \multirow{2}{*}{\multicolumn{2}{|c|}{$\begin{array}{c}2017 \\
(n=232)\end{array}$}} & \multirow{2}{*}{\multicolumn{2}{|c|}{$\begin{array}{c}\text { Total } \\
(n=4,140)\end{array}$}} \\
\hline & & & & & & & & & & & & \\
\hline & $\mathrm{n}$ & IR $\ddagger$ & $\mathrm{n}$ & IR & $\mathrm{n}$ & IR $\ddagger$ & $\mathrm{n}$ & IR $\ddagger$ & $\mathrm{n}$ & IR $\ddagger$ & $\mathrm{n}$ & IR $\ddagger$ \\
\hline Local pain & 65 & 1.06 & 60 & 0.58 & 305 & 0.66 & 14 & 0.03 & 7 & 0.02 & 451 & 0.28 \\
\hline Local erythema & 53 & 0.86 & 44 & 0.43 & 250 & 0.54 & 9 & 0.02 & 14 & 0.03 & 370 & 0.23 \\
\hline Local warmth & 53 & 0.86 & 43 & 0.42 & 247 & 0.53 & 5 & 0.01 & 4 & 0.01 & 352 & 0.22 \\
\hline Local swelling & 3 & 0.05 & 46 & 0.45 & 259 & 0.56 & 4 & 0.01 & 5 & 0.01 & 317 & 0.20 \\
\hline Fever & 48 & 0.78 & 66 & 0.64 & 156 & 0.34 & 19 & 0.04 & 9 & 0.02 & 298 & 0.19 \\
\hline Headache & 19 & 0.31 & 30 & 0.29 & 72 & 0.16 & 8 & 0.02 & 3 & 0.01 & 132 & 0.08 \\
\hline Rash & 7 & 0.11 & 12 & 0.12 & 84 & 0.18 & 5 & 0.01 & 1 & 0.00 & 109 & 0.07 \\
\hline Vomiting & 6 & 0.10 & 18 & 0.18 & 50 & 0.11 & 5 & 0.01 & 5 & 0.01 & 84 & 0.05 \\
\hline Pain & 4 & 0.07 & 29 & 0.28 & 34 & 0.07 & 12 & 0.02 & 4 & 0.01 & 83 & 0.05 \\
\hline Cough & 6 & 0.10 & 22 & 0.21 & 35 & 0.08 & 7 & 0.01 & 4 & 0.01 & 74 & 0.05 \\
\hline Local abscess & 5 & 0.08 & 15 & 0.15 & 52 & 0.11 & 0 & 0.00 & 1 & 0.00 & 73 & 0.05 \\
\hline Hypersensitivity & 22 & 0.36 & 10 & 0.10 & 26 & 0.06 & 1 & 0.00 & 3 & 0.01 & 62 & 0.04 \\
\hline Edema & 1 & 0.02 & 29 & 0.28 & 28 & 0.06 & 0 & 0.00 & 2 & 0.00 & 60 & 0.04 \\
\hline Myalgia & 7 & 0.11 & 10 & 0.10 & 35 & 0.08 & 2 & 0.00 & 2 & 0.00 & 56 & 0.04 \\
\hline Pruritus & 1 & 0.02 & 4 & 0.04 & 45 & 0.10 & 5 & 0.01 & 0 & 0.00 & 55 & 0.03 \\
\hline Local pruritus & 1 & 0.02 & 5 & 0.05 & 40 & 0.09 & 6 & 0.01 & 2 & 0.00 & 54 & 0.03 \\
\hline Influenza like illness & 11 & 0.18 & 29 & 0.28 & 5 & 0.01 & 6 & 0.01 & 1 & 0.00 & 52 & 0.03 \\
\hline Local induration & 30 & 0.49 & 14 & 0.14 & 4 & 0.01 & 1 & 0.00 & 3 & 0.01 & 52 & 0.03 \\
\hline Nausea & 1 & 0.02 & 15 & 0.15 & 28 & 0.06 & 1 & 0.00 & 6 & 0.01 & 51 & 0.03 \\
\hline Local nodule & 1 & 0.02 & 5 & 0.05 & 45 & 0.10 & 0 & 0.00 & 0 & 0.00 & 51 & 0.03 \\
\hline Dyspnea & 3 & 0.05 & 12 & 0.12 & 30 & 0.06 & 4 & 0.01 & 1 & 0.00 & 50 & 0.03 \\
\hline
\end{tabular}

†AEFI: Adverse Event Following Immunization; †IR: Incidence Rate per 100.000 doses of Instituto Butantan TIV (\#AEFI / \# doses)

with no SAE. The other 49 (31.6\%) notifications were received through spontaneous reports and from NIP, and among them, seven (14.3\%) pregnant women experienced at least one of the following SAE: diplegia, dyspnea, encephalitis, facial paralysis, hypotension, influenza like illness, myasthenic syndrome, neuropathy and rash.

Regarding the seven pregnant women who presented with SAE, two had full recovery and one pregnant woman with hypotension and dyspnea was hospitalized and died. For the other four pregnant women PV-IB did not receive any information on their outcomes. All these cases could not be assessed for causality due to insufficient information available to perform the evaluation and impossibility of contacting the reporters for further investigation (unauthorized follow-up by the reporter or absence of contact data).

\section{DISCUSSION}

This manuscript described the safety profile of the seasonal trivalent influenza vaccine manufactured by
Instituto Butantan from 2013 to 2017. The monitoring of AEFI performed through pharmacovigilance activities can provide useful information such as safety signals detected even if reports are incomplete ${ }^{10}$. According to AlguacilRamos et al..$^{10}$ post-licensing surveillance of AEFI is an integral part of the immunization programs.

In Brazil, the regulatory framework on "Pharmacovigilance Standards for Marketing Authorization Holders for Human Use" was established in 2009 by Brazilian Health Regulatory Agency (Anvisa) through the publication of Directive Resolution RDC no. 4 of 10/02/20094. This resolution, considered the pillar for the detection of safety signals through the identification of cases of adverse event after the use of a product, contributed to improve the pharmacovigilance activities not only for medicines but also for vaccines. Since then, MAH have made efforts to improve their ability to generate and detect new safety signals for the products included in the National Immunization Program (NIP).

In Brazil, public health services shall notify any AEFI to NIP. However, considering the MAH regulatory 
responsibility to monitor the product safety, PV-IB has maintained an open channel to receive any AEFI report directly from the vacinees or HCP from public health services. Besides this channel, NIP submit the AEFI notifications to PV-IB but there is no periodicity for sending the information.

As presented in the results section, in 2015 PV-IB received from NIP an exceptionally large amount of individual cases related to Instituto Butantan TIV. For the other years, the number of cases received was considerably smaller. Since PV-IB did not have access to individual information, and only had access to compiled data shared by NIP, no inferences could be made as to why the number of reported cases was so different that year compared to the other years' averages.

Unfortunately, the information received from NIP, especially those in the 2015 list, had insufficient details available so that it was not possible to proceed with the causality assessment. Most of those cases were considered "unclassifiable" with respect to the causality assessment. This was the reason for assessing few SAE regarding causality. The three SAE (arrhythmia, hypersensitivity and laryngeal edema) assessed were symptoms related to "anaphylactic reactions" that are predicted in the packet insert.

According to the Global Advisory Committee on Vaccine Safety of the World Health Organization ${ }^{11}$, pregnant women and infants are at increased risk for developing either complications or severe influenza-associated infection.

The Committee has also stated that there is robust information regarding the safe use of inactivated influenza vaccine in pregnant women, therefore, influenza immunization of this group should be a priority ${ }^{11,12}$.

The increasing exposure of this population to seasonal influenza TIV should be stressed, as pregnant women has been considered a target group for influenza vaccination by several immunization programs in high, middle and lowincome countries ${ }^{8,13}$. Immunization during pregnancy is not only safe, but has also contributed to reduce morbidity and mortality in pregnant women as well as in neonates and children under five years old. Influenza immunization of pregnant women has been practiced albeit the absence of classical clinical trials performed in that population. Because of that, post-licensing monitoring of the safety of that practice in pregnant women is an important pharmacovigilance activity ${ }^{14}$.

Overall, the frequency and types of AEFI with the Instituto Butantan TIV described in this manuscript demonstrate similar findings when compared to available data in the literature, including the rare occurrence of $\mathrm{GBS}^{8,15,16}$. According to our analysis, the incidence rate of GBS was 0.04 cases per 1 million doses distributed, while in literature the incidence rate is described as 1 case per million doses administered ${ }^{4}$.

Since the pharmacovigilance policies have been established, post-licensing surveillance became mandatory in Brazil and there has been a considerable improvement in detecting and addressing AEFI.

However, there are still some challenges to be overcome to further improve it such as the completeness of data collected. The lack of data compromises the overall data analysis and the prompt and adequate response to AEFI. Pharmacovigilance has been associated with underreporting of AEFI and somehow may have contributed to the lack of information. Unfortunately, healthcare professionals have not yet included AEFI notification as one of their priorities in their daily activities. Their perception of AEFI is less concerning that the VPD, which contributes to the missing data as well as to the underreporting of AEFI. Hazell and Shakir ${ }^{17}$ have identified, in a systematic review of the literature, a high occurrence of underreporting of adverse drug reactions in passive surveillance systems. A recommended strategy to improve coverage and the quality of the AEFI notification is the continuing education of the professionals involved ${ }^{17}$.

The data analysis performed by PV-IB could not show that the occurrence of unexpected AEFI had a causal relationship with TIV. The main reason was the impossibility for assessing causality for most of the AEFI due to the lack of information for the majority of ICSR.

An important limitation of this manuscript is how AEFI incidence rates were calculated since they were estimated based on the number of doses distributed instead of the number of applied doses. The amount of doses distributed by the manufacturer is usually greater than the applied doses and may underestimate the rate ${ }^{18}$.

According to Chen et al. ${ }^{19} \mathrm{WHO}$ and its partners developed a strategy to help Low- and Middle-Income Countries (LMIC) establish and strengthen their vaccine safety monitoring systems. The Global Vaccine Safety Blueprint ${ }^{20}$ (GVSB) proposes three strategic goals: (1) establish minimal vaccine safety capacity in all countries; (2) provide enhanced capacity when vaccines are manufactured in-country, or products are introduced in populations; and (3) provide a technical support network. GVSB implementation is ensured by a network of technical partners that participate in this initiative, offering a common platform to partners interested in furthering vaccine safety systems through the alignment of their activities, compiling them into a portfolio. The new challenge at this time is to develop active surveillance systems capable of better quality information on the risk of association between a vaccine administration and a potential AEFI. 
One key tool to improve the surveillance of AEFI, in order to obtain advances in the identification of safety signals, is the continuous training of health professionals working with immunization. Well-trained professionals to identify and investigate AEFI may improve the quality of collected data. The timely-manner collection of clinical and laboratory data may avoid misconclusions in the case causality assessment. Those misconclusions may lead to a decrease in the credibility of immunization programs ${ }^{21}$.

In conclusion, the consolidation of pharmacovigilance activities is an important strategy to ensure that AEFI are communicated in timely-manner by the immunization program to the population. These actions are important to maintain the population adherence to vaccination resulting in high vaccine coverage rates, and therefore, keeping control of vaccine-preventable diseases.

\section{CONFLICT OF INTERESTS}

The authors state that there is no conflict of interests.

\section{FUNDING}

No funding has been received.

\section{AUTHORS' CONTRIBUTIONS}

Vera L. Gattás, Patrícia Emília Braga, Marcelo E. Koike, Maria Beatriz B. Lucchesi, Mayra M. Moura de Oliveira, Roberta O Piorelli and Vivian Queiroz contributed with the study concept and design, data collection, analysis and interpretation of data and writing of the manuscript. Alexander Roberto Precioso participated designing the study and in the critical review of the intellectual content of the manuscript. All authors have approved the final version of the manuscript and are responsible for all aspects, ensuring its accuracy and completeness.

\section{REFERENCES}

1. World Health Organization. Influenza (Seasonal). [cited 2018 Nov 27]. Available from: http://www.who.int/mediacentre/ factsheets/fs211/en/

2. Asturias EJ, Wharton M, Pless R, MacDonald NE, Chen RT, Andrews N, et al. Contributions and challenges for worldwide vaccine safety: The Global Advisory Committee on Vaccine Safety at 15 years. Vaccine. 2016;34:3342-9.

3. Brasil. Ministério da Saúde. Informe técnico: $20^{\mathrm{a}}$ Campanha Nacional de Vacinação contra a Influenza. Brasília: Ministério da Saúde; 2018. [cited 2018 Nov 27]. Available from: http:// portalarquivos2.saude.gov.br/images/pdf/2018/abril/18/
Informe-Cp-Influenza---01-03-2018-Word-final-28.03.18\%20 final.pdf

4. Brasil. Agência Nacional de Vigilância Sanitária. Resolução $n^{\circ}$ 4, de 10 de fevereiro de 2009. Dispõe sobre as normas de f armacovigilância para os detentores de registro de medicamentos de uso humano. [cited 2018 Nov 27]. Available from: http://bvsms.saude.gov.br/bvs/saudelegis/anvisa/2009/ res0004_10_02_2009.html

5. MedDRA: Medical Dictionary for Regulatory Activities. [cited 2018 Mar 29]. Available from: http://www.meddra.org/

6. World Health Organization. Council for International Organizations of Medical Sciences. Definition and application of terms for vaccine pharmacovigilance: report of CIOMS/WHO Working Group on Vaccine Pharmacovigilance. Geneva: WHO; 2012. [cited 2018 Mar 29]. Available from: http://www.who.int/ vaccine_safety/initiative/tools/CIOMS_report_WG_vaccine. pdf

7. Uppsala Monitoring Centre. Glossary of pharmacovigilance terms. [cited 2018 Sep 20]. Available from: https://www.who-umc. org/global-pharmacovigilance/global-pharmacovigilance/ glossary

8. European Medicines Agency. Interim guidance on enhanced safety surveillance for seasonal influenza vaccines in the EU. London: EMA; 2014. [cited 2018 Nov 27]. Available from: https://www.ema.europa.eu/documents/scientific-guideline/ interim-guidance-enhanced-safety-surveillance-seasonalinfluenza-vaccines-eu_en-0.pdf

9. World Health Organization. Vaccines against influenza WHO position paper - November 2012. Wkly Epidemiol Rec. 2012;87:461-76.

10. Alguacil-Ramos AM, Muelas-Tirado J, Garrigues-Pelufo TM, Portero-Alonso A, Diez-Domingo J, Pastor-Villalba E, et al. Surveillance for adverse events following immunization (AEFI) for 7 years using a computerised vaccination system. Public Health. 2016;135:66-74.

11. World Health Organization. Global Advisory Committee on Vaccine Safety. Safety of immunization during pregnancy: a review of the evidence. Geneva: WHO; 2014. [cited 2018 Nov 27]. Available from: http://www.who.int/vaccine_safety/ publications/safety_pregnancy_nov2014.pdf

12. World Health Organization. SAGE Working Group. Background paper on influenza vaccines and immunization. [cited on 2018 Nov 27]. Available from: http://www.who.int/immunization/ sage/meetings/2012/april/1_Background_Paper_Mar26_v13_ cleaned.pdf

13. Kochhar S, Bauwens J, Bonhoeffer J.. Safety assessment of immunization in pregnancy. Vaccine. 2017;35:6469-71.

14. Bonhoeffer J, Kochhar S, Hirschfeld S, Heath PT, Jones CE, Bauwens J, et al. Global alignment of immunization safety assessment in pregnancy: the GAIA project. Vaccine. 2016;34:5993-7. 
15. Vellozzi C, Burwen DR, Dobardzic A, Ball R, Walton K, Haber P. Safety of trivalent inactivated influenza vaccines in adults: background for pandemic influenza vaccine safety monitoring. Vaccine. 2009;27:2114-20.

16. Vellozzi C, Broder KR, Haber P, Guh A, Nguyen M, Cano M, et al. Adverse events following influenza A (H1N1) 2009 monovalent vaccines reported to the Vaccine Adverse Event Reporting System, United States, October 1, 2009-January 31, 2010. Vaccine. 2010;28:7248-55.

17. Hazell L, Shakir SA. Under-reporting of adverse drug reactions: a systematic review. Drug Saf. 2006;29:385-96.

18. World Health Organization. Global manual on surveillance of adverse events following immunization. Geneva: WHO; 2014. [cited 2018 Nov 27]. Available from: http://www. who.int/vaccine_safety/publications/Global_Manual_on Surveillance_of_AEFI.pdf
19. Chen RT, Shimabukuro TT, Martin DB, Zuber PL, Weibel DM, Sturkenboom M. Enhancing vaccine safety capacity globally: a lifecycle perspective. Vaccine. 2015; 33 Suppl 4:D46-54.

20. World Health Organization. Department of Immunization, Vaccines and Biologicals. Global vaccine safety blueprint: the landscape analysis. Geneva: WHO; 2012. [cited 2018 Nov 27]. Available from: http://apps.who.int/iris/bitstream/ handle/10665/70854/WHO_IVB_12.04_eng.pdf?sequence=1

21. Gold MS, Balakrishnan MR, Amarasinghe A, MacDonald NE. An approach to death as an adverse event following immunization. Vaccine. 2016;34:212-7. 